\title{
Key role of congestion in natural history of heart failure
}

This article was published in the following Dove Press journal:

International Journal of General Medicine

I2 August 201 I

Number of times this article has been viewed

\section{Maya Guglin}

University of South Florida,

Tampa, FL, USA
Correspondence: Maya Guglin

University of South Florida,

Tampa, FL, USA

Tel +I 248346350

Email mguglin@gmail.com
Abstract: The natural course of heart failure with decreased and preserved systolic function is almost identical. The current concept of heart failure where decreased cardiac output plays the major role does not explain this similarity. We suggest a revised concept of heart failure where congestion plays the leading role. While congestion is almost invariably present in heart failure with normal and with reduced systolic function, the low output syndrome is only present in heart failure with reduced systolic function. The small difference in morbidity and mortality in favor of heart failure with preserved systolic function reflects the contribution of low output syndrome to the natural course of the disease. Congestion can result from low output or from multiple other conditions, but severity of congestion is the major determinant of progression of heart failure.

Keywords: heart failure, congestion, ejection fraction

\section{Background}

Heart failure is a final common pathway of practically all cardiac diseases, including coronary artery disease, hypertension, congenital cardiac defects, nonischemic cardiomyopathy, valvular defects of multiple etiologies, and infiltrative diseases. Surprisingly, the very term "heart failure" is not well defined. Multiple definitions circulating in the literature are inconsistent, and such inconsistency reflects the lack of uniform understanding of the essence of this condition. Figure 1 presents the classic chart of pathogenesis of heart failure. Decreased cardiac output triggers a cascade of neurohormonal activation that occupies center stage, with congestion being a modest byproduct of the chain of events. If this chart reflects the true picture, and decreased myocardial contractility plays the leading role, the mainstay of heart failure treatment should be inotropes. However, inotropes have a very limited place in management of heart failure. Besides, the chart does not apply to approximately one half of those patients with heart failure who have heart failure with preserved systolic function. These patients do not benefit from neurohormonal blockade, inotropes, ventricular assist devices, or cardiac transplantation. Most importantly, the natural course and prognosis in heart failure with preserved and with reduced systolic function is nearly identical. In other words, whether the pathogenetic mechanism in Figure 1 applies or not, the outcome is the same. There can be only one explanation, ie, decreased cardiac output does not play a key role in heart failure, and our current concept should be revised. 


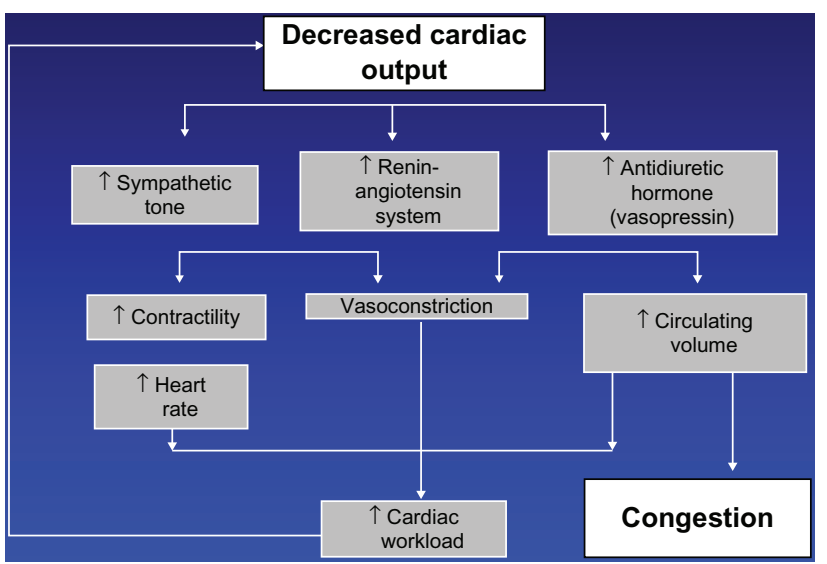

Figure I Current concept of heart failure.

\section{Guidelines on definition of heart failure}

The European Congress of Cardiology (2008) defines heart failure as a "syndrome in which the patients should have the following features: symptoms of HF, typically shortness of breath at rest or during exertion, and/or fatigue; signs of fluid retention such as pulmonary congestion or ankle swelling". ${ }^{1}$

The American College of Cardiology and the American Heart Association (2009) guidelines define heart failure as a "complex clinical syndrome ... . The cardinal manifestations of HF are dyspnea and fatigue, which may limit exercise tolerance, and fluid retention, which may lead to pulmonary congestion and peripheral edema". ${ }^{2}$

The Heart Failure Society of America (2010) defines heart failure as "a syndrome caused by cardiac dysfunction ... whether the dysfunction is primarily systolic or diastolic or mixed, it leads to neurohormonal and circulatory abnormalities, usually resulting in characteristic symptoms such as fluid retention, shortness of breath, and fatigue, especially on exertion". ${ }^{3}$

In summary, all definitions agree that heart failure is a syndrome that clinically manifests itself as congestion. This part of all the above texts is strong and clear and obviously does not cause any controversies. The second part of all the definitions is vague and controversial.

According to the American College of Cardiology and the American Heart Association definition, the syndrome of congestion "... can result from any structural or functional cardiac disorder that impairs the ability of the ventricle to fill with or eject blood". ${ }^{2}$ The ability of the ventricle to fill with blood reflects its diastolic function. The ability of the ventricle to eject blood reflects its systolic function.
Systolic function is measured by left ventricular ejection fraction using echocardiography or other imaging modalities, or by cardiac output using a Swan-Ganz catheter. There is plenty of evidence that $40 \%-50 \%$ of patients with heart failure have normal systolic function. It was once believed that although left ventricular ejection fraction is preserved, "effective blood flow" can be impaired. ${ }^{4}$ However, several studies have demonstrated that blood flow is normal or increased in a substantial number of patients with heart failure with preserved systolic function. ${ }^{5,6}$

Diastolic function, or left ventricular filling pressure, is evaluated by echocardiography or by Swan-Ganz catheter. There is very little evidence that diastolic function is normal in any substantial proportion of the heart failure population. In heart failure patients with both reduced and with preserved left ventricular ejection fraction, diastolic dysfunction by echocardiography was present in more than $90 \%$ of cases. ${ }^{7}$ None of the heart failure patients with ejection fraction less than $35 \%$ had normal diastolic function. ${ }^{8}$ In the Olmstead County heart failure cohort, only $10 \%$ of patients with preserved ejection fraction and $5 \%$ of patients with reduced ejection fraction had normal diastolic function. ${ }^{9}$ In another cohort, only one in 45 participants with heart failure had normal diastolic function.

Diastolic dysfunction, unlike systolic dysfunction, reflects severity of congestion. One of the parameters of diastolic function, namely the $\mathrm{E} / \mathrm{e}^{\prime}$ ratio, correlates closely with intracardiac filling pressures. ${ }^{10-17}$ Simple volume overload with intravenous saline creates gradual progression of diastolic filling pattern from normal to restrictive. ${ }^{18}$

While systolic dysfunction may or may not be present in heart failure, diastolic dysfunction is mandatory. Diastolic dysfunction is the equivalent of congestion. Diastolic dysfunction is the common denominator of heart failure. There is no heart failure without congestion. There is no heart failure without diastolic dysfunction. Diastolic function may improve and become normal as a result of treatment or natural evolution of the disease, but if there is no congestion, no diastolic dysfunction, and no elevated left ventricular end diastolic pressure at any time, there is no basis for a diagnosis of heart failure. Therefore, it is incorrect to state that heart failure results from either/or the inability of the ventricle to fill with or eject blood. It always results from the inability of the ventricle to fill with blood, while the function of ejection may be normal or impaired. Inability of the left ventricle to eject blood is important to the degree of the severity of congestion resulting from it. 
According to the Heart Failure Society of America definition, the syndrome of congestion is "caused by cardiac dysfunction, generally resulting from myocardial muscle dysfunction or loss and characterized by either LV dilation or hypertrophy or both. Whether the dysfunction is primarily systolic or diastolic or mixed, it leads to neurohormonal and circulatory abnormalities ...". 3

The issue with primarily systolic or diastolic dysfunction or mixed is analyzed above. Neurohormonal abnormalities are well documented in heart failure with systolic dysfunction but not with preserved systolic function. All attempts to treat heart failure with preserved systolic function using medications targeting neurohormonal changes have failed. The CHARM-Preserved (Candesartan in Heart Failure Assessment of Reduction in Mortality and Morbidity) trial randomized 3023 patients between candesartan and placebo ${ }^{19}$ and failed to demonstrate a significant effect on cardiovascular death, but fewer heart failure hospitalizations in the candesartan-treated patients were observed. These results are difficult to interpret because the cutoff of left ventricular ejection fraction in the CHARM trial was $40 \%$, which creates a mix of heart failure with normal systolic function and with mild systolic dysfunction. The PEP-CHF (Perindopril in Elderly People with Chronic Heart Failure) study showed no effect of perindopril on mortality and heart failure hospitalizations. ${ }^{20}$ The I-PRESERVE (Irbesartan in HF with preserved ejection fraction) trial did not demonstrate any mortality or morbidity benefit from irbesartan. ${ }^{21} \mathrm{~A}$ recent meta-analysis ${ }^{22}$ combined these studies to increase their statistical power and also failed to demonstrate a significant effect of inhibition of the renin-angiotensin-aldosterone system on mortality in preserved systolic function.

The statement of left ventricular dilatation or hypertrophy is also soft. The left ventricle may be dilated, but it may be normal in size. It can be hypertrophied but may also have perfectly normal wall thickness.

To summarize, heart failure is a clinical syndrome of congestion. It may be caused by decreased systolic function or other conditions causing volume overload. Therefore, evidence of congestion should be mandatory for the diagnosis of heart failure.

\section{Guidelines on evidence of congestion}

According to ADHERE (the Acute Decompensated Heart Failure National Registry), which included patients with and without left ventricular systolic dysfunction, most patients admitted for heart failure are "wet" or congested, with dyspnea, rales, edema, radiological signs of fluid overload, or combination of the above. ${ }^{23}$ However, making the diagnosis of heart failure with preserved systolic function by only clinical criteria and normal ejection fraction could result in overdiagnosis of this condition. In a study by Caruana et al, ${ }^{24}$ most patients with normal ejection fraction who were diagnosed as having heart failure in fact suffered from other diseases, such as coronary artery disease, obesity, chronic obstructive pulmonary disease, or other conditions explaining their symptoms, and only seven of 109 had heart failure.

To diagnose heart failure with preserved systolic function accurately, one has to demonstrate evidence of increased intracardiac pressures. Vasan and Levy proposed standardized clinical criteria where the diagnosis of "definite" heart failure requires clinical heart failure and ejection fraction $\geq 50 \%$ assessed within 72 hours after diagnosis and confirmed elevated filling pressures. ${ }^{25}$ The timing requirement was later found to be unnecessary, because it was shown that ejection fraction does not change during decompensation and remains relatively stable. ${ }^{26}$ The Working Group on Myocardial Function of the European Society of Cardiology ${ }^{27}$ required evidence of diastolic dysfunction (pulmonary capillary wedge pressure $>12 \mathrm{mmHg}$ or left ventricular end diastolic pressures $>16 \mathrm{mmHg}$ ) provided by cardiac catheterization or by Doppler velocities on echocardiography.

According to the European Society of Cardiology, three obligatory conditions are needed for the diagnosis of heart failure with normal systolic function. They include the presence of signs or symptoms of congestive heart failure, ejection fraction $>50 \%$, and evidence of left ventricular diastolic dysfunction. ${ }^{28}$

Unfortunately, the majority of studies enrolling patients with heart failure and normal systolic function did not have documentation of either intracardiac pressure or left ventricular diastolic dysfunction. More importantly, not all current guidelines require rigorous assessment of diastolic dysfunction to establish a diagnosis of heart failure. Meanwhile, because congestion is the essence of heart failure, whether with preserved or reduced systolic function, evidence of congestion should be a mandatory part of the assessment of patients who may have heart failure based on symptoms. Addressing the requirements for initial assessment of patients with heart failure, American College of Cardiology and the American Heart Association guidelines recommend echocardiography for assessment of “... left ventricular ejection fraction, left ventricular size, wall 
thickness, and valve function ..." but do not even mention diastolic dysfunction. ${ }^{2}$

The easiest way to estimate intracardiac pressures is to measure the level of brain natriuretic peptide, but in the American College of Cardiology and the American Heart Association guidelines it is recommended only as a supplemental tool "to improve diagnostic accuracy". ${ }^{2}$ Amazingly, recommended initial laboratory evaluation of patients presenting with heart failure includes complete blood count, urinalysis, serum electrolytes (including calcium and magnesium), blood urea nitrogen, serum creatinine, fasting blood glucose, lipid profile, liver function tests, and thyroid-stimulating hormone, but not brain natriuretic peptide. ${ }^{2}$ In fact, brain natriuretic peptide is far more important for establishing the diagnosis of heart failure than the lipid profile, thyroid hormones, or any other listed parameters. On the contrary, the Heart Failure Society of America guidelines recommend measurement of brain natriuretic peptide in every patient with suspected heart failure. ${ }^{3}$ Similarly, European guidelines include brain natriuretic peptide as a mandatory step in establishing the diagnosis of heart failure. ${ }^{1}$

In summary, if all guidelines agree that heart failure is a syndrome of congestion, then evidence of congestion and assessment of its severity should be a cornerstone of the diagnosis and ongoing patient evaluation on follow-up. This applies similarly to heart failure with normal and reduced systolic function.

\section{Low output syndrome}

It is impossible to deny that not only congestion but also low output plays an important role in the natural history of heart failure, but only in heart failure with reduced systolic function. Multiple studies have reported decreased left ventricular ejection fraction as a poor predictive sign. ${ }^{29,30}$ In order to quantify its role and to compare it with the role of congestion, we have to compare the course of heart failure with normal and decreased systolic function.

Patients with preserved heart failure are usually older, more frequently women, have less coronary disease and myocardial infarction, and have more atrial fibrillation and other comorbidities. They have higher systolic blood pressures and pulse pressures, ${ }^{29}$ as well as a higher prevalence of left ventricular hypertrophy, aortic valve disease, and anemia. $^{30}$

Despite these dissimilarities, the reported mortality is either similar ${ }^{30-34}$ or somewhat better in preserved systolic function. ${ }^{35,36}$ Earlier studies published in the 1980s and early 1990s reported a better prognosis in preserved ejection fraction, but more recent studies have identified no differences in mortality. ${ }^{37}$

Patients with heart failure with both reduced and normal systolic function have congestion, but only patients with reduced systolic function have low output. Therefore, the difference in morbidity and mortality between heart failure with preserved and reduced left ventricular ejection fraction reflects the contribution of low output syndrome to the natural course of heart failure. Similarities in the natural course of heart failure with reduced and with preserved systolic function are likely explained by the syndrome of congestion shared by both groups. Using therapies unique for heart failure with the component of low output, or left ventricular remodeling, or reduced left ventricular systolic dysfunction (beta-blockers, angiotensin-converting enzyme inhibitors, cardiac resynchronization therapy, implantable cardioverter-defibrillator devices, ventricular assist devices, and cardiac transplantation) we are closing the small gap between morbidity and mortality in heart failure with normal and decreased systolic function. To treat the syndrome of congestion, which determines the course of heart failure to a much greater degree than the syndrome of low output, we use diuretics. The role of diuretics in the treatment of heart failure is discussed elsewhere. ${ }^{38,39}$ It typically takes fewer than 100 patients and several weeks of follow-up to realize that patients with heart failure cannot live without them, ${ }^{40-43}$ while it took thousands of patients and years of follow-up to demonstrate the survival benefit of angiotensin-converting enzyme inhibitors or beta-blockers. ${ }^{44,45}$

It is difficult to imagine a patient with heart failure who has never experienced congestion. However, there are clearly symptomatic patients with heart failure who are not congested. They are a small subset of very sick patients with low output and low systolic blood pressure. In full agreement with the pathogenetic chart (Figure 1), they benefit from inotropes, an intra-aortic balloon pump, any means of mechanical circulation, and cardiac transplantation.

Inotropes were not proven to be beneficial in any of the randomized controlled trials, but everybody uses them empirically. Even if such patients are congested, they can rarely be diuresed without inotropes. The OPTIME-HF (Outcomes of Prospective Trial of Intravenous Milrinone for Acute Exacerbation of Chronic Heart Failure) trial unfortunately was not very informative because patients requiring inotropic support were not included. ${ }^{46}$ 


\section{Revised concept of heart failure}

In the pathogenetic scheme reflecting the true relationship of two major syndromes in heart failure, ie, congestion, which is mandatory, and low output, which is optional, congestion occupies central place (Figure 2).

Congestion not only causes symptoms of volume overload, but it also determines the prognosis. ${ }^{47}$ Congestion causes cardiorenal syndrome, ${ }^{48,49}$ pulmonary hypertension, and right ventricular failure. All of these symptoms exacerbate the problem, causing further congestion. Because congestion plays the central role, diuretics are the only group of medications equally effective in heart failure with preserved or reduced systolic function. In inpatient or outpatient settings, in systolic or diastolic heart failure, diuretics invariably remain the top prescribed drugs. ${ }^{23}$

In about $50 \%$ of patients with heart failure, congestion is caused by left ventricular systolic dysfunction. It has been shown that heart failure with preserved and reduced systolic function is typically not different stages of the same disease but rather separate entities. The left ventricular ejection fraction has a bimodal distribution. ${ }^{50}$

However, the relationship between intracardiac pressure and ejection fraction may be more complex. Evidence suggests that persistence of congestion or high intracardiac pressures results in decreased contractility, and decongestion improves contractility. In animal experiments, myocardial edema results in an immediate decrease in contractility. ${ }^{51}$ Aggressive diuresis resulted not only in decreased pulmonary capillary wedge pressure but also in an increase in left ventricular ejection fraction from $35.7 \%$ to $39.5 \%$ in a matter of six days. ${ }^{52}$

Even electrical instability, which is usually a feature of left ventricular remodeling, may be precipitated by congestion. Studies of tracings from implantable devices with OptiVol $^{\circledR}$ indicate there is a weak but significant association between decreased intrathoracic impedance and malignant ventricular arrhythmias. ${ }^{53,54}$

The other $50 \%$ of patients with heart failure who have normal systolic function have congestion resulting from other causes, including renal dysfunction, infiltrative diseases of the myocardium, iatrogenic fluid overload, ischemia, hypertension with left ventricular hypertrophy, or any other conditions increasing intravascular volume, or altering myocardial relaxation. They demonstrate less electrical instability, are less prone to sudden cardiac death, and benefit little from inhibition of renin-angiotensin or beta-blockade.

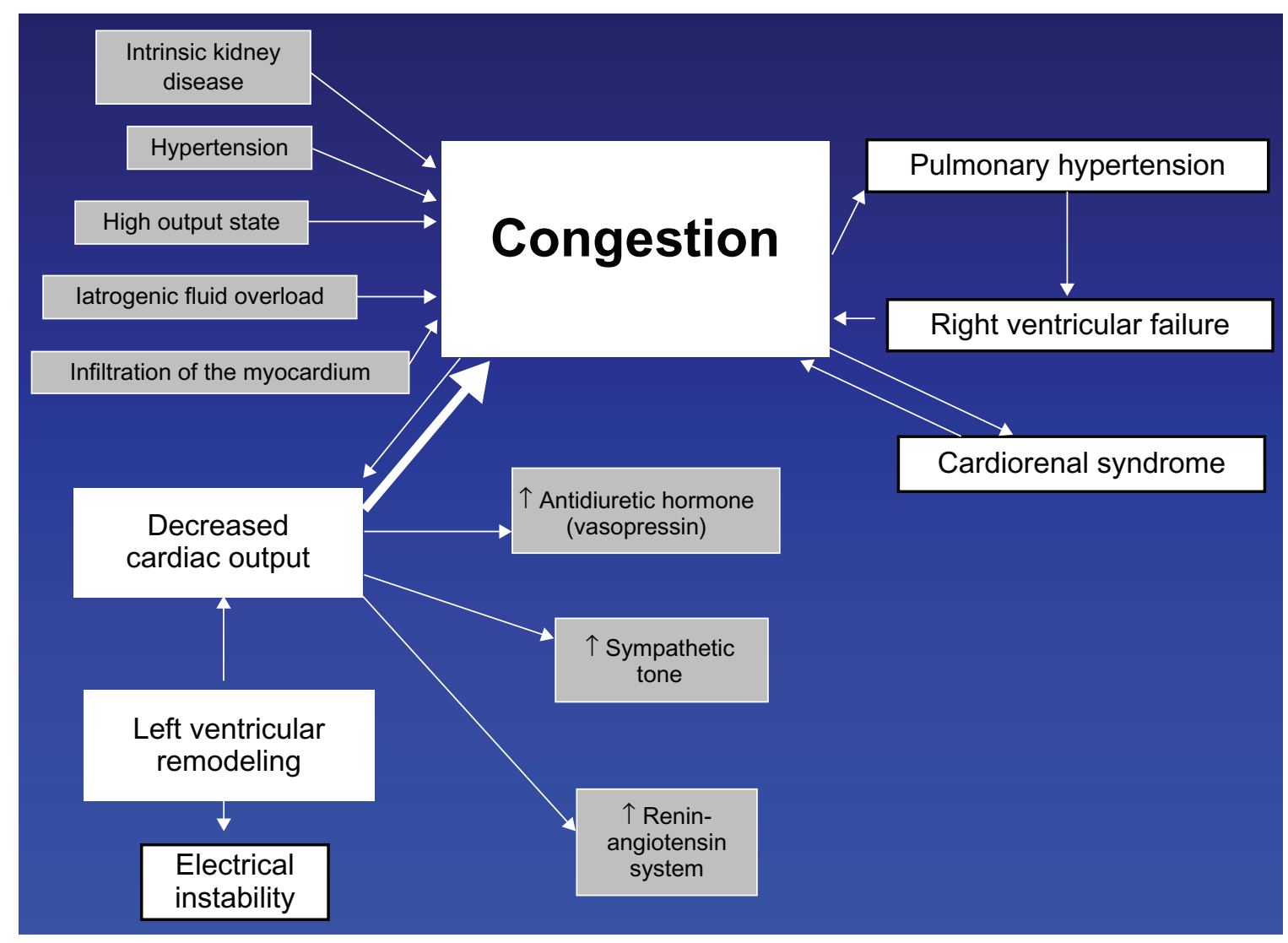

Figure 2 Suggested concept of heart failure. 


\section{Conclusion}

Heart failure is a syndrome of congestion resulting from decreased left ventricular systolic function or any other condition causing fluid retention or altering relaxation of the myocardium. Evidence of congestion, increased intracardiac pressures, or diastolic dysfunction is a mandatory step in the initial diagnosis of heart failure, and evaluation of severity of congestion is a mandatory step in all subsequent evaluation of patients with heart failure. Congestion is the main factor that determines the natural course of heart failure. The role of low output syndrome, or decreased systolic function, can be defined as the difference between morbidity and mortality in heart failure with reduced and with preserved systolic function. Guidelines that currently define the strategy of diagnosis and management of heart failure could benefit from revisions consistent with this concept.

\section{Disclosure}

The author reports no conflicts of interest in this work.

\section{References}

1. Dickstein K, Cohen-Solal A, Filippatos G, et al. ESC Guidelines for the diagnosis and treatment of acute and chronic heart failure 2008: The Task Force for the Diagnosis and Treatment of Acute and Chronic Heart Failure 2008 of the European Society of Cardiology. Developed in collaboration with the Heart Failure Association of the ESC (HFA) and endorsed by the European Society of Intensive Care Medicine (ESICM). Eur Heart J. 2008;29(19):2388-2442.

2. Hunt SA, Abraham WT, Chin MH, et al. 2009 Focused update incorporated into the ACC/AHA 2005 Guidelines for the Diagnosis and Management of Heart Failure in Adults. A Report of the American College of Cardiology Foundation/American Heart Association Task Force on Practice Guidelines Developed in Collaboration With the International Society for Heart and Lung Transplantation. J Am Coll Cardiol. 2009;53(15):e1-e90.

3. Lindenfeld J, Albert NM, Boehmer JP, et al. HFSA 2010 Comprehensive Heart Failure Practice Guideline. J Card Fail. 2010;16(6):e1-e194.

4. Andrew P. Diastolic heart failure demystified. Chest. 2003;124(2): 744-753.

5. Maurer MS, King DL, El-Khoury Rumbarger L, Packer M, Burkhoff D. Left heart failure with a normal ejection fraction: Identification of different pathophysiologic mechanisms. J Card Fail. 2005;11(3):177-187.

6. Maurer MS, Burkhoff D, Fried LP, Gottdiener J, King DL, Kitzman DW. Ventricular structure and function in hypertensive participants with heart failure and a normal ejection fraction: The Cardiovascular Health Study. J Am Coll Cardiol. 2007;49(9):972-981.

7. Brucks S, Little WC, Chao T, et al. Contribution of left ventricular diastolic dysfunction to heart failure regardless of ejection fraction. Am J Cardiol. 2005;95(5):603-606.

8. Samad BA, Olson JM, Alam M. Characteristics of left ventricular diastolic function in patients with systolic heart failure: A Doppler tissue imaging study. J Am Soc Echocardiogr. 2005;18(9):896-900.

9. Bursi F, Weston SA, Redfield MM, et al. Systolic and diastolic heart failure in the community. JAMA. 2006;296(18):2209-2216.

10. Ommen SR, Nishimura RA, Appleton CP, et al. Clinical utility of Doppler echocardiography and tissue Doppler imaging in the estimation of left ventricular filling pressures: A comparative simultaneous Doppler-catheterization study. Circulation. 2000;102(15):1788-1794.
11. Lester SJ, Tajik AJ, Nishimura RA, Oh JK, Khandheria BK, Seward JB. Unlocking the mysteries of diastolic function: Deciphering the Rosetta Stone 10 years later. J Am Coll Cardiol. 2008;51(7):679-689.

12. Liang HY, Cauduro SA, Pellikka PA, et al. Comparison of usefulness of echocardiographic Doppler variables to left ventricular end-diastolic pressure in predicting future heart failure events. Am J Cardiol. 2006;97(6):866-871.

13. Maurer MS, Packer M, Burkhoff D. Diastolic heart failure. $N$ Engl J Med. 2004;351(11):1143-1145.

14. Maurer MS, Spevack D, Burkhoff D, Kronzon I. Diastolic dysfunction: Can it be diagnosed by Doppler echocardiography? J Am Coll Cardiol. 2004;44(8):1543-1549.

15. Nishimura RA, Abel MD, Hatle LK, et al. Significance of Doppler indices of diastolic filling of the left ventricle: Comparison with invasive hemodynamics in a canine model. Am Heart J. 1989;118(6): $1248-1258$.

16. Nishimura RA, Appleton CP, Redfield MM, Ilstrup DM, Holmes DR Jr, Tajik AJ. Noninvasive Doppler echocardiographic evaluation of left ventricular filling pressures in patients with cardiomyopathies: A simultaneous Doppler echocardiographic and cardiac catheterization study. J Am Coll Cardiol. 1996;28(5):1226-1233.

17. Nishimura RA, Tajik AJ. Evaluation of diastolic filling of left ventricle in health and disease: Doppler echocardiography is the clinician's Rosetta Stone. J Am Coll Cardiol. 1997;30(1):8-18.

18. Masutani S, Little WC, Hasegawa H, Cheng HJ, Cheng CP. Restrictive left ventricular filling pattern does not result from increased left atrial pressure alone. Circulation. 2008;117(12):1550-1554.

19. Yusuf S, Pfeffer MA, Swedberg K, et al. Effects of candesartan in patients with chronic heart failure and preserved left-ventricular ejection fraction: The CHARM-Preserved Trial. Lancet. 2003;362(9386):777-781.

20. Cleland JG, Tendera M, Adamus J, Freemantle N, Polonski L, Taylor J. The perindopril in elderly people with chronic heart failure (PEP-CHF) study. Eur Heart J. 2006;27(19):2338-2345.

21. Massie BM, Carson PE, McMurray JJ, et al. Irbesartan in patients with heart failure and preserved ejection fraction. $N \mathrm{Engl} \mathrm{J} \mathrm{Med}$. 2008;359(23):2456-2467

22. Shah RV, Desai AS, Givertz MM. The effect of renin-angiotensin system inhibitors on mortality and heart failure hospitalization in patients with heart failure and preserved ejection fraction: A systematic review and meta-analysis. J Card Fail. 2010;16(3):260-267.

23. Yancy CW, Fonarow GC, Committee ASA. Quality of care and outcomes in acute decompensated heart failure: The ADHERE registry. Curr Heart Fail Rep. 2004;1(3):121-128.

24. Caruana L, Petrie MC, Davie AP, McMurray JJ. Do patients with suspected heart failure and preserved left ventricular systolic function suffer from "diastolic heart failure" or from misdiagnosis? A prospective descriptive study. BMJ. 2000;321(7255):215-218.

25. Vasan RS, Levy D. Defining diastolic heart failure: A call for standardized diagnostic criteria. Circulation. 2000;101(17):2118-2121.

26. Gandhi SK, Powers JC, Nomeir AM, et al. The pathogenesis of acute pulmonary edema associated with hypertension. $N$ Engl J Med. 2001;344(1):17-22.

27. [No authors listed]. How to diagnose diastolic heart failure. European Study Group on Diastolic Heart Failure. Eur Heart J. 1998;19(7):990-1003.

28. Paulus WJ, Tschope C, Sanderson JE, et al. How to diagnose diastolic heart failure: A consensus statement on the diagnosis of heart failure with normal left ventricular ejection fraction by the Heart Failure and Echocardiography Associations of the European Society of Cardiology. Eur Heart J. 2007;28(20):2539-2550.

29. Ansari M, Alexander M, Tutar A, Massie BM. Incident cases of heart failure in a community cohort: Importance and outcomes of patients with preserved systolic function. Am Heart J. 2003;146(1): 115-120.

30. Berry C, Hogg K, Norrie J, Stevenson K, Brett M, McMurray J. Heart failure with preserved left ventricular systolic function: A hospital cohort study. Heart. 2005;91(7):907-913. 
31. Bhatia RS, Tu JV, Lee DS, et al. Outcome of heart failure with preserved ejection fraction in a population-based study. $N$ Engl J Med. 2006;355(3):260-269.

32. Varela-Roman A, Gonzalez-Juanatey JR, Basante P, et al. Clinical characteristics and prognosis of hospitalised inpatients with heart failure and preserved or reduced left ventricular ejection fraction. Heart. 2002;88(3):249-254.

33. Varela-Roman A, Grigorian L, Barge E, Bassante P, de la Pena MG, Gonzalez-Juanatey JR. Heart failure in patients with preserved and deteriorated left ventricular ejection fraction. Heart. 2005;91(4):489-494

34. Fonarow GC, Stough WG, Abraham WT, et al. Characteristics, treatments, and outcomes of patients with preserved systolic function hospitalized for heart failure: A report from the OPTIMIZE-HF Registry. J Am Coll Cardiol. 2007;50(8):768-777.

35. Vasan RS, Larson MG, Benjamin EJ, Evans JC, Reiss CK, Levy D. Congestive heart failure in subjects with normal versus reduced left ventricular ejection fraction: Prevalence and mortality in a populationbased cohort. J Am Coll Cardiol. 1999;33(7):1948-1955.

36. Sweitzer NK, Lopatin M, Yancy CW, Mills RM, Stevenson LW. Comparison of clinical features and outcomes of patients hospitalized with heart failure and normal ejection fraction $(\geq 55 \%)$ versus those with mildly reduced ( $40 \%$ to $55 \%$ ) and moderately to severely reduced (<40\%) fractions. Am J Cardiol. 2008;101(8):1151-1156.

37. Senni M, Redfield MM. Heart failure with preserved systolic function. A different natural history? J Am Coll Cardiol. 2001;38(5):1277-1282.

38. Guglin M. Diuretics as pathogenetic treatment for heart failure. Int $J$ Gen Med. 2011;4:91-98.

39. Guglin M. Reappraisal of the role of diuretics in heart failure. Cardiol Rev. 2009;17(2):56-59.

40. Richardson A, Bayliss J, Scriven AJ, Parameshwar J, Poole-Wilson PA, Sutton GC. Double-blind comparison of captopril alone against frusemide plus amiloride in mild heart failure. Lancet. 1987;2(8561):709-711.

41. Cowley AJ, Stainer K, Wynne RD, Rowley JM, Hampton JR. Symptomatic assessment of patients with heart failure: Double-blind comparison of increasing doses of diuretics and captopril in moderate heart failure. Lancet. 1986;2(8510):770-772.

42. van Kraaij DJ, Jansen RW, Bruijns E, Gribnau FW, Hoefnagels WH. Diuretic usage and withdrawal patterns in a Dutch geriatric patient population. J Am Geriatr Soc. 1997;45(8):918-922.

43. Mathur PN, Pugsley SO, Powles AC, McEwan MP, Campbell EJ. Effect of diuretics on cardiopulmonary performance in severe chronic airflow obstruction. A controlled clinical trial. Arch Intern Med. 1984;144(11):2154-2157.
44. [No authors listed]. Effect of enalapril on survival in patients with reduced left ventricular ejection fractions and congestive heart failure. The SOLVD Investigators. N Engl J Med. 1991;325(5):293-302.

45. Hjalmarson A, Goldstein S, Fagerberg B, et al. Effects of controlledrelease metoprolol on total mortality, hospitalizations, and well-being in patients with heart failure: The Metoprolol CR/XL Randomized Intervention Trial in congestive heart failure (MERIT-HF). MERIT-HF Study Group. JAMA. 2000;283(10):1295-1302.

46. Cuffe MS, Califf RM, Adams KF Jr, et al. Short-term intravenous milrinone for acute exacerbation of chronic heart failure: A randomized controlled trial. JAMA. 2002;287(12):1541-1547.

47. Lee D AP, Stukel T, Alter D, Chong A, Parker J, Tu J. "Dose-dependent" impact of recurrent cardiac events on mortality in patients with heart failure. Am J Med. 2009;122:162.e161-e162.

48. Guglin M, Rivero A, Matar F, Garcia M. Renal dysfunction in heart failure is due to congestion but not low output. Clin Cardiol. 2011;34(2):113-116.

49. Mullens W, Abrahams Z, Francis GS, et al. Importance of venous congestion for worsening of renal function in advanced decompensated heart failure. J Am Coll Cardiol. 2009;53(7):589-596.

50. Owan TE, Hodge DO, Herges RM, Jacobsen SJ, Roger VL, Redfield MM. Trends in prevalence and outcome of heart failure with preserved ejection fraction. $N$ Engl J Med. 2006;355(3):251-259.

51. Rubboli A, Sobotka PA, Euler DE. Effect of acute edema on left ventricular function and coronary vascular resistance in the isolated rat heart. Am J Physiol. 1994;267(3 Pt 2):H1054-H1061.

52. Parrinello G, Paterna S, Di Pasquale P, et al. Changes in estimating echocardiography pulmonary capillary wedge pressure after hypersaline plus furosemide versus furosemide alone in decompensated heart failure. J Card Fail. 2011;17(4):331-339.

53. Ip JE, Cheung JW, Park D, et al. Temporal associations between thoracic volume overload and malignant ventricular arrhythmias: A study of intrathoracic impedance. J Cardiovasc Electrophysiol. 2011;22(3):293-299.

54. Moore HJ, Peters MN, Franz MR, Karasik PE, Singh SN, Fletcher RD Intrathoracic impedance preceding ventricular tachyarrhythmia episodes. Pacing Clin Electrophysiol. 2010;33(8):960-966.
International Journal of General Medicine

\section{Publish your work in this journal}

The International Journal of General Medicine is an international, peer-reviewed open-access journal that focuses on general and internal medicine, pathogenesis, epidemiology, diagnosis, monitoring and treatment protocols. The journal is characterized by the rapid reporting of reviews, original research and clinical studies across all disease areas.

\section{Dovepress}

A key focus is the elucidation of disease processes and management protocols resulting in improved outcomes for the patient. The manuscript management system is completely online and includes a very quick and fair peer-review system. Visit http://www.dovepress.com/ testimonials.php to read real quotes from published authors. 